Tropical Journal of Pharmaceutical Research May 2021; 20 (5): 893-898

ISSN: $1596-5996$ (print); 1596-9827 (electronic)

(C) Pharmacotherapy Group, Faculty of Pharmacy, University of Benin, Benin City, 300001 Nigeria.

\title{
Tetramethyl pyrazine exerts anti-apoptotic and antioxidant effects in a mouse model of MPTP-induced Parkinson's disease via regulation of the expressions of Bax, Bcl-2, Nrf2 and GCLC
}

\author{
Lixing Dai ${ }^{1}$, Ruiqing Diao², Jinghua Zhang ${ }^{3}$, Mengying Cao${ }^{1}$, Hongli Gao ${ }^{4}$, Bibo \\ Tang ${ }^{5 *}$ \\ ${ }^{1}$ Department of General Practice, Hubei Province Third People's Hospital Affiliated to Jianghan University, Wuhan 430033, \\ ${ }^{2}$ Department of Neurology, Gucheng County Hospital of Hebei Province, Hengshui 253800, Hebei Province, ${ }^{3}$ Department of \\ Encephalopathy, Tianjin Beichen District Hospital of Traditional Chinese Medicine, Tianjin 300400, ${ }^{4}$ Department of Medical, \\ Hubei Minzu University, Enshi 445000, ${ }^{5}$ Department of Critical Care Medicine, Hubei Province Third People's Hospital Affiliated \\ to Jianghan University, Wuhan 430033, Hubei Province, China
}

*For correspondence: Email: tzkf64@163.com

Sent for review: 19 January 2021

Revised accepted: 26 April 2021

\begin{abstract}
Purpose: To investigate the effect of tetramethyl pyrazine (TMP) on MPTP)-mediated neuronal apoptosis and oxidative imbalance in mice, and the mechanism of action involved.

Methods: Forty-five mice were assigned evenly to blank control, MPTP and TMP groups. The protein concentrations of Bax, Bcl-2, cytochrome C (Cyt c), Nrf2, GCLC and cleaved caspase-3; and levels of glutathione (GSH) and thiobarbituric acid reactive products (TBARS) were evaluated and compared amongst the groups.

Results: Cyt c, Bax, and cleaved caspase-3 protein levels in TMP group were significantly lower than those in MPTP group, while Bcl-2 protein expression was higher in TMP group than in MPTP mice ( $p<$ 0.05). Furthermore, TBARS was lower in TMP group than in MPTP group, while GSH level increased, relative to MPTP mice. The levels of Nrf2 and GCLC were significantly higher in TMP group than in MPTP group $(p<0.05)$.

Conclusion: Tetramethyl pyrazine exerts anti-apoptotic and antioxidant effects on MPTP-mediated Parkinsonism via regulation of the expressions of Bax, Bcl-2, Nrf2 and glutamate-cysteine ligase catalytic subunit. Thus, TMP has potential for use in the treatment Parkinson's disease.
\end{abstract}

Keywords: Tetramethyl pyrazine, Bax, Bcl-2, Nrf2, glutamate-cysteine ligase catalytic subunit, MPTP, Parkinsonism

This is an Open Access article that uses a fund-ing model which does not charge readers or their institutions for access and distributed under the terms of the Creative Commons Attribution License (http://creativecommons.org/licenses/by/4.0) and the Budapest Open Access Initiative (http://www.budapestopenaccessinitiative.org/read), which permit unrestricted use, distribution, and reproduction in any medium, provided the original work is properly credited.

Tropical Journal of Pharmaceutical Research is indexed by Science Citation Index (SciSearch), Scopus, International Pharmaceutical Abstract, Chemical Abstracts, Embase, Index Copernicus, EBSCO, African Index Medicus, JournalSeek, Journal Citation Reports/Science Edition, Directory of Open Access Journals (DOAJ), African Journal Online, Bioline International, Open-J-Gate and Pharmacy Abstracts

\section{INTRODUCTION}

Parkinson's disease (PD) is predominant in middle-aged and elderly people. The disease manifests in symptoms such as static tremor, enhanced muscular tone, and delayed movement, all of which seriously affect the patient's quality of life [1]. Epidemiological reports show that, as a result of the increase in ageing population in China, the incidence of PD 
is increasing year by year. Thus, Parkinson's disease has become a serious health problem in the field of chronic diseases of the elderly in China [2].

At present, the pathogenesis of PD is not fully understood. Thus, clinically, there is no effective radical cure for the disease. Usually, drug intervention is used to delay the progression of the disease. However, long-term drug intervention may cause many adverse reactions, in addition to the fact that efficacy of the longterm treatment is not satisfactory [3]. Therefore, it is important to identify and develop new drugs with ideal clinical efficacy and high reliability for treatment of PD patients.

Tetramethyl pyrazine (TMP) is extracted from the rhizome of Ligusticum chuanxiong Hort. It is used in the treatment of systemic diseases such as diseases of the heart, brain and central nervous system. In addition, studies have shown that it exerts anti-tumor and anti-fibrotic effects $[4,5]$.

Previous investigations have shown that the expression level of Bax in substantia nigra neurons is significantly increased in PD [6]. Other studies have confirmed that Bax ablation and $\mathrm{Bcl}-2$ overexpression protect the neurons from MPTP-induced damage [7]. It is known that Nrf2 performs effective anti-oxidative stress functions in a wide range of living organisms [8]. Studies have demonstrated that glutamate-cysteine ligase catalytic subunit (GCLc) ameliorates oxidative stress-induced liver injury due to various factors [9]. However, at present, there are limited reports on the effect of TMP on levels Bax, Bcl-2, Nrf2, and GCLc in PD. The present study was carried out to investigate the effect of TMP on MPTP-induced neuronal cell apoptosis and oxidative stress in a mouse model of PD, and the involvement of $\mathrm{Bax}, \mathrm{Bcl}-2, \mathrm{Nrf} 2$, and GCLc in the process.

\section{EXPERIMENTAL}

\section{Materials}

Forty-five male Wistar mice were purchased from Changsha Tianqin Biotechnology Co. Ltd (batch number: SCXK 2019-0005). The mice were raised in a greenhouse and allowed ad libitum access feed and drinking water. All protocols used in this study were approved by Animal Protection and Use Committee of The Fourth Military Medical University (Wuhan, China, approval no. 201903891) and conduct of the studies followed the guidelines of "Principles of Laboratory Animal Care" (NIH publication no. 8523, revised 1985) [10].

\section{Main reagents and instruments}

HPLC equipment was purchased from Nanjing Kejie Analytical Instrument Co. Ltd. Confocal laser microscope was bought from Beijing Xinshengyuan Biomedical Technology Co. Ltd. Electronic Analytical Balance was product of Kebang Xingye (Beijing) Technology Co. Ltd. High-speed electric homogenizer was purchased from Suzhou Abitto Biotechnology Co. Ltd. Vortex oscillator was obtained from Wuxi Microchromatography Biotechnology Co. Ltd, while High speed centrifuge was purchased from Sichuan Shuke Instrument Co. Ltd.

Monoclonal antibodies for Bax and Bcl-2 were purchased from Shanghai Guangrui Biotechnology Co. Ltd. Cytochrome C was bought from Wuhan Ipu Biotechnology Co. Ltd, while Nrf2 monoclonal antibody was purchased from Shanghai Seg Biotechnology Co. Ltd. Tyrosine hydroxylase was supplied by Beijing Baiolaibo Technology Co. Ltd. Caspase-3 was purchased from lacoin (Wuhan) Biotechnology Co. Ltd, while MPTP was produced by Jinclun (Beijing) Biotechnology Co. Ltd. Tetramethyl pyrazine (TMP) was purchased from Shanghai Hengfei Biotechnology Co. Ltd, while GCLc monoclonal antibody was obtained from Xiamen Huijia Biotechnology Co. Ltd.

\section{Animal studies}

Forty-five (45) mice were assigned to blank control, MPTP and TMP groups, each with 15 mice. Animals in TMP group received intraperitoneal (i.v.) injection of TMP at a dose of $20 \mathrm{mg} / \mathrm{kg}$ once per day for 7 consecutive days. Mice in the TMP group were injected with MPTP ( $1 \mu \mathrm{mol}$ MPTP dissolved in $2 \mu \mathrm{L}$ of normal saline) in the substantia nigra on day 7 , and then TMP administration was done for 7 days. Mice in blank control and MPTP groups were given equivalent volume of normal saline i.v. once daily for 7 consecutive days. The MPTP group received MPTP injection in the substantia nigra for 7 days. After 14 days, mice in each group were sacrificed and their striata and substantia nigra were isolated for subsequent studies.

Flow cytometry was employed to measure the apoptotic ability of substantia nigra cells in each group. The substantia nigra cells were routinely digested with $0.25 \%$ trypsin and centrifuged at $1000 \mathrm{r} / \mathrm{min}$ for $10 \mathrm{~min}$. After discarding the supernatant, the cells were resuspended in PBS solution and adjusted to a concentration of $1 \times 10^{6}$ cells $/ \mathrm{mL}$, followed by centrifugation again at 1000 $\mathrm{r} / \mathrm{min}$ for $5 \mathrm{~min}$, and resuspension of the cells in binding buffer. Thereafter, $5 \mu \mathrm{L}$ of Annexin V- 
FITC was added, followed by thorough mixing and incubation at laboratory temperature for 30 min. After centrifugation, the supernatant was discarded, and the cells were resuspended in binding buffer. Then, $10 \mu \mathrm{L}$ of propidium iodide was added to mix well, placed at room temperature $\left(25{ }^{\circ} \mathrm{C}\right)$ for $30 \mathrm{~min}$, and finally subjected to flow cytometry. Apoptosis (A) was computed as shown in Eq 1.

$A(\%)=(T a / T c) 100$

where $\mathrm{Ta}$ and $\mathrm{Tc}$ are the total number of apoptotic and normal cells, respectively.

Relative protein amounts of Bax, Bcl-2, Cyt c, Nrf2, GCLc and cleaved caspase-3 in the substantia nigra of mice in each group were detected with western blot assay. Total protein of substantia nigra cells was extracted and its concentration was determined with BCA protein quantitative kit. After separation by SDS gel electrophoresis, the protein was electrically transferred to PVDF membrane. After sealing and immune reaction, chemiluminescence chromogenic agent was added for color development. Finally, the corresponding completed image was used for copy processing.

The levels of oxidative stress related indexes glutathione (GSH) and thiobarbituric acid reactive substances (TBARS) in the substantia nigra of mice in each group were detected with colorimetric method and chemiluminescence method, respectively. Colorimetric method: test tubes were taken and labeled as measuring tube, non-enzyme tube, blank tube and duplicating tube respectively. $0.2 \mathrm{~mL}$ of $\mathrm{GSH}$ was added to all tubes except blank control group. $0.2 \mathrm{~mL}$ of serum to be detected was added to the measuring tube, and $0.2 \mathrm{~mL}$ of double distilled water was added to the other tubes. After mixing well, the tubes were placed in a water bath for 5 $\min$ at $37^{\circ} \mathrm{C}$. Then, $0.1 \mathrm{~mL}$ of $\mathrm{H}_{2} \mathrm{O}_{2}$ was added to all tubes except the blank tube. After incubating in a water bath for $5 \mathrm{~min}, 0.8 \mathrm{~mL}$ of phosphoric acid precipitant was added to the blank tube, and $2 \mathrm{~mL}$ of phosphoric acid precipitant was added to the other tubes. After centrifugation, supernatants were taken, $1.25 \mathrm{~mL}$ of disodium hydrogen phosphate and $0.25 \mathrm{~mL}$ of DTNB were added, respectively, and detected at $422 \mathrm{~nm}$ wavelength. Chemiluminescence method: $1 \%$ sample was placed in the measuring tube, and equal amount of distilled water was put into the control measuring tube. $1 \mathrm{~mL}$ of reagent $1,0.1 \mathrm{~mL}$ of reagent $2,0.1 \mathrm{~mL}$ of reagent 3 and $0.1 \mathrm{~mL}$ of reagent 4 were put into each tube respectively. After fully mixing, the sample was placed in a constant temperature water bath at $37^{\circ} \mathrm{C}$ for
$20 \mathrm{~min}$. $2 \mathrm{~mL}$ of chromogenic reagent was added to each measuring tube, mixed well and put it at room temperature for $20 \mathrm{~min}$. The measuring tubes were placed in a $1 \mathrm{~cm}$ light-diameter colorimetric cup, adjusted to zero with distilled water, and colorimetric reaction was carried out at $550 \mathrm{~nm}$. The content of TBARS in each group was calculated according to the kit instructions.

\section{Statistical analysis}

Levels of TBARS and GSH in mice in each group are presented as mean \pm SD. Two-group comparison was carried out with Student's $t$-test, while single factor ANOVA was used for multigroup comparisons. All statistical analyses were done with SPSS version 23.0, and significant difference was assumed at $p<0.05$.

\section{RESULTS}

\section{Effect of TMP on apoptosis of PD mice}

The percentage apoptosis was markedly increased in MPTP group, relative to control, but percentage apoptosis was markedly decreased in TMP group, relative to MPTP group. These results are presented on Table 1.

Table 1: Level of apoptosis in the mice (mean \pm SD, $n$ $=15$ )

\begin{tabular}{ll}
\hline Group & Apoptosis (\%) \\
\hline Blank control & $5.01 \pm 0.78$ \\
MPTP & $9.07 \pm 1.40^{\mathrm{a}}$ \\
TMP & $5.23 \pm 0.69^{\mathrm{b}}$ \\
\hline${ }^{\mathrm{a} P}<0.05$, vs control; ${ }^{\mathrm{b}} p<0.05$, vs MPTP mice
\end{tabular}

\section{Effect of TMP on apoptosis-related proteins in PD mice}

The relative protein concentrations of Cyt c, Bax, and cleaved caspase-3 in MPTP group were significantly higher than the corresponding expression levels in blank control group, while $\mathrm{Bcl}-2$ protein was lower in MPTP group than in control. However, there were lower concentrations of these proteins in TMP group than in MPTP group, while the expression level of $\mathrm{Bcl}-2$ was markedly higher in TMP group. These results are presented on Table 2 and Figure 1.

\section{Effect of TMP on oxidative stress in PD mice}

As shown in Table 3, the TBARS level in TMP group was markedly raised, relative to control, while GSH was markedly reduced in the control group. In contrast, TBARS level was significantly higher in the TMP group than in the MPTP group while GSH level was lower.

Trop J Pharm Res, May 2021; 20(5): 895 
Table 2: Comparison of apoptosis in PD mice (mean \pm $\mathrm{SD}, \mathrm{n}=15$ )

\begin{tabular}{|c|c|c|c|c|}
\hline Group & Bax & $\mathrm{Bcl}-2$ & Cyt C & $\begin{array}{c}\text { Cleaved } \\
\text { caspase-3 }\end{array}$ \\
\hline \multirow{2}{*}{$\begin{array}{l}\text { Blank } \\
\text { control }\end{array}$} & $0.31 \pm 0$ & $0.35 \pm 0$ & $1.00 \pm 0$ & \\
\hline & $\begin{array}{c}.06 \\
0.69 \pm 0\end{array}$ & $\begin{array}{c}.05 \\
0.03 \pm 0\end{array}$ & $\begin{array}{c}.01 \\
1.49 \pm 0\end{array}$ & $0.19 \pm 0.02$ \\
\hline \multirow[t]{2}{*}{ MPTP } & $.12^{\mathrm{a}}$ & $.01^{\mathrm{a}}$ & $.09^{\mathrm{a}}$ & $1.38 \pm 0.09^{a}$ \\
\hline & $0.45 \pm 0$ & $0.31 \pm 0$ & $1.05 \pm 0$ & \\
\hline TMP & $.08^{\mathrm{b}}$ & $.03^{\mathrm{b}}$ & $.05^{\mathrm{b}}$ & $0.25 \pm 0.05^{\mathrm{b}}$ \\
\hline \multicolumn{5}{|c|}{${ }^{a} P<0.05$, vs control; ${ }^{b} p<0.05$, vs MPTP group } \\
\hline A & B & $\mathbf{C}$ & & \\
\hline \multicolumn{2}{|c|}{ - $=$} & - & $\operatorname{Bax}$ & \\
\hline \multicolumn{2}{|c|}{ 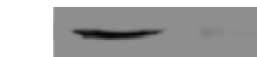 } & & BC1-2 & \\
\hline \multicolumn{3}{|c|}{$\longrightarrow$} & \multicolumn{2}{|c|}{ Cylochrome e } \\
\hline \multicolumn{3}{|c|}{ 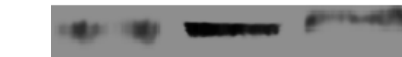 } & \multicolumn{2}{|c|}{ Cleayage of Cespese-3 } \\
\hline & & & Bactin & \\
\hline
\end{tabular}

Figure 1: Comparison of protein expression levels of apoptosis-related indicators in PD mice cells. Key: A: Blank control group; B: MPTP group; C: TMP group

Table 3: Levels of TBARS and GSH in the mice (mean $\pm \mathrm{SD}, \mathrm{n}=15$ )

\begin{tabular}{lcc}
\hline Group & TBARS $(\mathbf{n m o l} / \mathbf{m g})$ & GSH $(\mathbf{n m o l} / \mathbf{m g})$ \\
\hline MPTP & $3.75 \pm 0.15$ & $12.36 \pm 1.15$ \\
TMP & $6.98 \pm 1.05^{\mathrm{a}}$ & $6.98 \pm 1.36^{\mathrm{a}}$ \\
Blank control & $4.63 \pm 0.11^{\mathrm{b}}$ & $9.45 \pm 1.16^{\mathrm{b}}$ \\
\hline a $P<0.05$, vs control ${ }^{\mathrm{b}} \mathrm{p}<0.05$, vs MPTP mice
\end{tabular}

\section{Effect of TMP on protein expression levels of Nrf2 and GCLc in PD mice}

The protein expression levels of Nrf2 and GCLc in PD mice were raised in MPTP group, relative to blank control. However, protein expression levels of Nrf2 and GCLc in PD mice were lower in TMP group than in MPTP group. These results are presented in Table 4 and Figure 2.

Table 4: Protein expression levels of Nrf2 and GCLc in each group

\begin{tabular}{lcc}
\hline Group & Nrf2 & GCLC \\
\hline MPTP & $1.00 \pm 0.20$ & $0.20 \pm 0.02$ \\
TMP & $0.13 \pm 0.06^{\mathrm{a}}$ & $0.05 \pm 0.01^{\mathrm{a}}$ \\
Blank control & $0.45 \pm 0.14^{\mathrm{b}}$ & $0.19 \pm 0.03^{\mathrm{b}}$ \\
\hline${ }^{\mathrm{a} P}<0.05$, vs control; ${ }^{\mathrm{b}} p<0.05$, vs MPTP group
\end{tabular}

\section{DISCUSSION}

Previous reports have shown that TMP exerts neuroprotective effects in vivo and in vitro. In addition, TMP has been shown to play a protective role against ischemic brain injury, and to accelerate cell proliferation and differentiation [11]. It is known that MPTP is a neurotoxin that induces Parkinson's disease via its effect on neurons, and it has been widely used in the establishment of animal models of the disease [12]. However, there are no extant studies on the potential of TMP to block apoptosis of neurons in PD through anti-oxidant effects. This formed the basis of the present study.

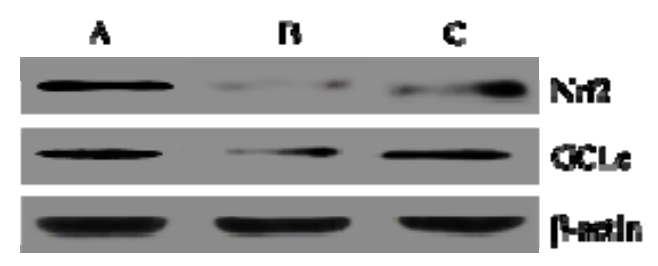

Figure 2: Comparison of protein expression levels of Nrf2 and GCLC in PD mice in each group. Key: A: Blank control; B: MPTP, and C: TMP groups

The Bax protein is apoptosis-inducing. In contrast, $\mathrm{Bcl}-2$ is an anti-apoptosis protein that blocks Bax translocation and dimer production and inhibits Cyt c release, thereby blocking apoptosis $[13,14]$. Several studies have shown that caspase- 3 plays a key role in apoptosisrelated processes. In this study, apoptosisrelated changes in the substantia nigra was determined, and it was revealed that Cyt $\mathrm{c}$ and Bax protein expressions were significantly raised in MPTP group, while Bcl-2 protein expression was significantly downregulated when caspase-3 was cleaved. Thus, the MPTP-induced changes in the above indices were effectively blocked by TMP. These results suggest that TMP effectively inhibits MPTP-induced apoptosis of rat brain substantia nigra cells [15]. Clinical studies have demonstrated that oxidative damage plays a key role in osteoporotic and dopamine-neuron attenuation-related processes which accelerate mitochondrial apoptosis, thereby aggravating oxidative damage and triggering "oxidative stress-cell apoptosis" cycle.

Other reports have shown that MPTP accentuates release free radicals, while triggering attenuation of dopamine neurons and oxidative stress response. Many drugs inhibit MPTP-induced neurotoxicity by eliminating free radicals. The release of TBARS reflects the degree of oxidative damage in vivo. Reduced glutathione (GSH) is an important reductant that participates in resistance to oxidative damage in the brain. It participates in the scavenging of reactive oxygen radicals and parent-child electronic reagents, which is of great significance in metabolic processes. The levels of GSH are significantly reduced in PD patients and MPTPinduced animal models [16]. 
In the present study, the effect of TMP and MPTP on redox balance in the substantia nigra of mice was determined, and it was shown that TBARS levels were significantly decreased, while GSH levels were significantly increased in the MPTP group, indicating the occurrence of oxidative damage. However, TMP intervention effectively blocked the increase in TBARS and decrease in GSH. In vivo, Nrf2 is the strongest anti-oxidative stress regulatory transcription factor which activates antioxidant enzymes, including GCLc in cell redox regulation, thereby protecting the cells from damage by reactive oxygen species.

Finally, the potential molecular mechanism involved in the protective effect of TMP against MPTP-induced redox imbalance was investigated. The MPTP-induced downregulations of Nrf2 and GCLc were effectively blocked after TMP intervention. This suggests strongly that TMP blocks MPTP-induced oxidative stress injury in substantia nigra of PD mice.

\section{CONCLUSION}

The results obtained in this investigation indicate that TMP exerts anti-apoptotic and anti-oxidative effects on MPTP-damaged neurons in a mouse Parkinsonism model through regulation of expression levels of Bax, Bcl-2, Nrf2, and GCLc. Therefore, TMP can potentially be developed for the management of Parkinson's disease.

\section{DECLARATIONS}

\section{Conflict of interest}

No conflict of interest is associated with this work.

\section{Contribution of authors}

We declare that this work was performed by the authors named in this article and all liabilities pertaining to claims relating to the content of this article will be borne by the authors. Bibo Tang designed the study, supervised the data collection, and analyzed the data. Lixing Dai interpreted the data and prepared the manuscript for publication. Ruiqing Diao, Jinghua Zhang, Mengying Cao and Hongli Gao supervised the data collection, analyzed the data and reviewed the draft of the manuscript.

\section{Open Access}

This is an Open Access article that uses a fund- ing model which does not charge readers or their institutions for access and distributed under the terms of the Creative Commons Attribution License (http://creativecommons.org/licenses/by/ 4.0) and the Budapest Open Access Initiative (http://www.budapestopenaccessinitiative.org/rea d), which permit unrestricted use, distribution, and reproduction in any medium, provided the original work is properly credited.

\section{REFERENCES}

1. Zhan ZW, Lin LZ, Yu EH, Xin JW, Lin L, Lin $H L, Y e Q Y$, Chen XC, Pan XD. Abnormal resting-state functional connectivity in posterior cingulate cortex of Parkinson's disease with mild cognitive impairment and dementia. CNS Neurosci Ther 2018; 24(10): 897-905.

2. Walter J, Bolognin S, Antony PMA, Nickels SL, Poovathingal SK, Salamanca L, Magni S, Perfeito $R$, Hoel F, Qing X, et al. Neural Stem Cells of Parkinson's Disease Patients Exhibit Aberrant Mitochondrial Morphology and Functionality. Stem Cell Reports 2019; 12(5): 878-889.

3. Feng $P$, Zhang X, Li D, Ji C, Yuan Z, Wang R, Xue G, Li $G$, Holscher C. Two novel dual GLP-1/GIP receptor agonists are neuroprotective in the MPTP mouse model of Parkinson's disease. Neuropharmacol 2018; 133: 385-394.

4. Zhang C, Shen M, Teng F, Li P, Gao F, Tu J, Luo L, Yeh CK, Zhang D. Ultrasound-Enhanced Protective Effect of Tetramethylpyrazine via the ROS/HIF-1A Signaling Pathway in an in Vitro Cerebral Ischemia/Reperfusion Injury Model. Ultrasound Med Biol 2018; 44(8): 17861798.

5. Raghu R, Karthikeyan S. Zidovudine and isoniazid induced liver toxicity and oxidative stress: Evaluation of mitigating properties of silibinin. Environ Toxicol Pharmacol 2016; 46: 217-226.

6. Haddadi $R$, Nayebi AM, Eyvari Brooshghalan $S$. Silymarin prevents apoptosis through inhibiting the Bax/caspase-3 expression and suppresses toll like receptor-4 pathway in the SNc of 6-OHDA intoxicated rats. Biomed Pharmacother 2018; 104: 127-136.

7. Hu M, Li F, Wang W. Vitexin protects dopaminergic neurons in MPTP-induced Parkinson's disease through PI3KJAkt signaling pathway. Drug Des Devel Ther 2018; 12: 565-573.

8. Hou Y, Wang Y, He Q, Li L, Xie H, Zhao Y, Zhao J. Nrf2 inhibits NLRP3 inflammasome activation through regulating Trx1/TXNIP complex in cerebral ischemia reperfusion injury. Behav Brain Res 2018; 336: 32-39.

9. Mao Z, Li S, Zhang L, Chen M. The mTOR/GCLc/GSH Pathway Mediates the Dose-Dependent Bidirectional Regulation of ROS Induced by TiO 2 NPs in Neurogenic Cells. Oxi Med Cellular longevity, 2019, 2019(9):1-14.

10. World Health Organization. Principles of laboratory animal care. WHO Chron 1985; 39: 51-56. 
11. Mehmood K, Zhang H, Li K, Wang L, Rehman MU, Nabi $F$, lqbal MK, Luo $H$, Shahzad M, Li J. Effect of tetramethylpyrazine on tibial dyschondroplasia incidence, tibial angiogenesis, performance and characteristics via HIF-1a/NEGF signaling pathway in chickens. Sci Rep 2018; 8(1): 2495.

12. Zhu P, Hu S, Jin Q, Li D, Tian F, Toan S, Li Y, Zhou H, Chen Y. Ripk3 promotes ER stress-induced necroptosis in cardiac IR injury: A mechanism involving calcium overload/XO/ROS/mPTP pathway. Redox Biol 2018; 16 : 157-168.

13. YYu S, Gong LS, Li NF, Pan YF, Zhang L. Galangin (GG) combined with cisplatin (DDP) to suppress human lung cancer by inhibition of STAT3-regulated NF-KB and Bcl2/Bax signaling pathways. Biomed Pharmacother 2018; 97: 213-224.
14. Wei L, Chen Q, Guo A, Fan J, Wang R, Zhang H. Asiatic acid attenuates CCl4-induced liver fibrosis in rats by regulating the PI3KJAKT/mTOR and Bcl-2/Bax signaling pathways. Int Immunopharmacol 2018; 60: 1-8.

15. Bergandi L, Mungo E, Morone R, Bosco O, Rolando B, Doublier S. Hyperglycemia Promotes Chemoresistance Through the Reduction of the Mitochondrial DNA Damage, the Bax/Bcl-2 and Bax/Bcl-XL Ratio, and the Cells in Sub-G1 Phase Due to Antitumoral Drugs Induced-Cytotoxicity in Human Colon Adenocarcinoma Cells. Front Pharmacol 2018; 9: 866.

16. Zhou H, Li D, Zhu P, Ma Q, Toan S, Wang J, Hu S, Chen $Y$, Zhang $Y$. Inhibitory effect of melatonin on necroptosis via repressing the Ripk3-PGAM5-CypD-mPTP pathway attenuates cardiac microvascular ischemia-reperfusion injury. J Pineal Res 2018; 65(3): e12503. 